\title{
PD-L1 Expression in TNBC: A Predictive Biomarker of Response to Neoadjuvant Chemotherapy?
}

\author{
Bruna Cerbelli, ${ }^{1}$ Angelina Pernazza, ${ }^{1}$ Andrea Botticelli, ${ }^{2}$ \\ Lucio Fortunato, ${ }^{3}$ Massimo Monti, ${ }^{4}$ Paolo Sciattella, ${ }^{5}$ Domenico Campagna, ${ }^{6}$ \\ Federica Mazzuca, ${ }^{2}$ Maria Mauri, ${ }^{7}$ Giuseppe Naso, ${ }^{8}$ Paolo Marchetti, ${ }^{2}$ \\ Giulia d'Amati, ${ }^{1}$ and Leopoldo Costarelli' \\ ${ }^{1}$ Department of Radiological, Oncological and Pathological Sciences, Sapienza, University of Rome, Rome, Italy \\ ${ }^{2}$ Oncology Unit, Sant'Andrea Hospital, Sapienza University of Rome, Rome, Italy \\ ${ }^{3}$ Department of Surgery, San Giovanni-Addolorata, Rome, Italy \\ ${ }^{4}$ Department of Surgical Sciences, Sapienza University of Rome, Rome, Italy \\ ${ }^{5}$ Department of Statistical Sciences, Sapienza University of Rome, Rome, Italy \\ ${ }^{6}$ Department of Pathology, San Giovanni-Addolorata, Rome, Italy \\ ${ }^{7}$ Department of Oncology, San Giovanni-Addolorata, Rome, Italy \\ ${ }^{8}$ Oncology Unit, Sapienza University of Rome, Rome, Italy
}

Correspondence should be addressed to Giulia d’Amati; giulia.damati@uniromal.it

Bruna Cerbelli and Angelina Pernazza contributed equally to this work.

Received 8 August 2017; Accepted 19 November 2017; Published 14 December 2017

Academic Editor: Maria L. Gasparri

Copyright (C) 2017 Bruna Cerbelli et al. This is an open access article distributed under the Creative Commons Attribution License, which permits unrestricted use, distribution, and reproduction in any medium, provided the original work is properly cited.

Triple negative breast cancer (TNBC) has an aggressive clinical behaviour, with a poorer prognosis compared to other subtypes. Recently, tumor-infiltrating lymphocytes (TILs) have been proposed as a predictive biomarker for a better clinical outcome and pathological response (pR) after neoadjuvant chemotherapy (NACT) in TNBC. These data confirm the role of the immune system in the neoplastic progression and in the response to therapy. We performed a retrospective analysis of 54 pre-NACT biopsies of TNBC and compared both the percentage of stromal TILs and the degree of PD-L1 expression with the extent of pR to standard NACT. A pathological complete response (pCR) was achieved in $35 \%$ of cases. Univariate analysis showed (i) a significant association between PD-L1 expression in $\geq 25 \%$ of neoplastic cells and the achievement of a pCR ( $p=0.024$ ); (ii) a significantly higher frequency of pCR in cases showing $\geq 50 \%$ stromal TILs $(p<0.001)$. However in the multivariate analysis only PD-L1 expression on tumor cells remained significantly associated with pCR $(\mathrm{OR}=1,13$; $95 \% \mathrm{CI} 1,01-1,27)$, suggesting that the expression of this biomarker could be associated with a subpopulation of TNBC more likely to respond to chemotherapy. These data need to be confirmed by larger studies.

\section{Introduction}

Triple negative breast cancer (TNBC) accounts for 10-20\% of all breast cancers [1]. It is often associated with high histological grade, presence of lymphocytic infiltration, high rate of distant metastasis, and a poorer prognosis when compared to other breast cancer subtypes. TNBC is generally treated with standard chemotherapy regimens, including both anthracyclines and taxanes, either in the metastatic, adjuvant, or neoadjuvant setting. Neoadjuvant chemotherapy [NACT] is increasingly used in the management of this $\mathrm{BC}$ subtype, with pathologic complete response (pCR) rate ranging from $30 \%$ to $50 \%$ [2-4]. These data point to the need of biomarkers that could be useful to identify the subset of patients more prone to achieve a pCR. In recent reports the presence of tumor-infiltrating lymphocytes (TILs) has been shown to predict the response of TNBC to NACT [57]. Moreover, a high number of stromal TILs is predictive 
of a more favorable outcome in this BC subset. These data underscore the crucial role of the immune system both in the neoplastic progression and in the response to therapy and support the robustness of biomarkers of tumor-immune system interplay in clinical practice [8]. The interaction between programmed cell death protein 1 (PD-1) and its ligand (PD-L1) represents a mechanism of immune escape and a therapeutic target for poor-prognosis malignancies, such as melanoma and non-small-cell lung cancer (NSCLC) [9]. PD-1 is a transmembrane protein of $40 \mathrm{kDa}$ expressed on CD8+ and CD4+ T cells, natural killer (NK) cells, B cells, activated monocytes, and dendritic cells [10]. It is a negative regulator of the immune system that functions by forming a complex with its ligands (either PDL1 or PDL2). Only limited and contrasting data on the role of PD-L1 in breast cancer have been reported so far. In fact, the expression of this marker has been correlated with either a worst [11] or a better prognosis [12].

Apart from their prognostic or predictive value, the presence of stromal TILs and the expression of PD-L1 are strong markers of immune activation in breast cancer and could be involved in the response to preoperative systemic treatment. In this study we aimed to investigate the role of PD-L1 expression and stromal TILs in predicting the pathological response to NACT in TNBC. We retrospectively analyzed 54 pre-NACT biopsies and compared both the percentage of stromal TILs and the extent of PD-L1 expression on neoplastic and inflammatory cells with the effect of neoadjuvant chemotherapy.

\section{Materials and Methods}

Between January 2011 and December 2016, 54 consecutive patients with TNBC received standard NACT (4 cycles of doxorubicin + cyclophosphamide Q3W followed by 12 cycles of paclitaxel weekly) at our Institutions. Clinical information, including age, clinical stage at diagnosis, type of surgery, and pathologic response, was extracted from the institutional databases.

2.1. Evaluation of Stromal Tumor-Infiltrating Lymphocytes. Pre-therapy biopsies were retrieved from the Pathology Departments at the Sapienza University Teaching Hospital and the San Giovanni-Addolorata Hospital. Hematoxylineosin stained slides were blindly re-evaluated for the presence of stromal tumor-infiltrating lymphocytes (TILs) according to a previously published method [13]. Briefly, TILs were quantified as a percentage of the stromal area of the tumor and expressed as a continuous parameter.

\subsection{Evaluation of PD-L1 Expression and Immunophenotyping} of the Inflammatory Infiltrate. Serial sections were obtained from each paraffin block for (i) immunohistochemical evaluation of PD-L1 expression on both neoplastic and inflammatory cells and (ii) immunophenotyping of the inflammatory infiltrate. PD-L1 immunostains were performed with one of the antibody clones approved for diagnostic assay (SP142, rabbit IgG, dilution 1:200, catalog \#M4420; Spring
Bioscience, Pleasanton, CA) [14] at 1:100 dilution, using an automated immunostainer (Benchmark XT, Ventana Medical System, Tucson, AZ, USA) with the Optiview DAB IHC detection kit (Ventana Medical Systems, Tucson, Arizona, USA) according to manufacturer's instructions. Relevant positive controls (human tonsils and placenta) were used for each run of staining. Negative controls were obtained by omitting the primary antibody. The expression of PD-L1 was evaluated separately on all tumor cells and inflammatory infiltrates. A minimum of 200 neoplastic cells were present in each biopsy sample. A positive stain was defined as the presence of membrane staining, either strong or weak, complete or incomplete, in a percentage of cells $\geq 1 \%$, that is, the threshold reported for clinical response to PD-L1 inhibitors in nonsmall-cell lung carcinoma and has also been reported in breast carcinoma $[15,16]$. For each biopsy, both the intensity of membrane staining (scored as $1+$ weak, $2+$ moderate, and $3+$ strong) and the percentage of positive neoplastic cells were recorded, while only the percentage of positive inflammatory cells was evaluated.

Immunophenotyping of the inflammatory infiltrates was carried out with the following antibodies: CD3 for T lymphocytes (Roche, 1:100); CD4 (1:40) for the helper T subset; CD8 for the cytotoxic T subset (1:100); CD20 for B lymphocytes (1:200) CD68 for macrophages $(1: 100)$, and N-CAM $(1: 100)$ (all from Novocastra, Newcastle, UK). Four images at 20x original magnification (accounting for one $\mathrm{mm}^{2}$ of tumor field) were acquired from the areas of maximum inflammatory infiltrate by the NIS Elements Viewer mounted on a Zeiss Axioskop 2 microscope. The number of positive cells $/ \mathrm{mm}^{2}$ for each antibody was then manually counted on the acquired images.

2.3. Evaluation of the Pathologic Response to NACT. The degree of pathologic response of each patient to NACT was obtained from the pathology reports. A complete response was defined as the complete disappearance of invasive tumor cells from breast tissue and regional lymph nodes, regardless of the presence of residual ductal carcinoma in situ (ypT0/is, ypNo) $[17,18]$.

2.4. Statistical Analysis. In the descriptive analysis, quantitative variables were described as mean and range, while qualitative variables were reported as number and percentage. Univariate associations between clinicopathological features and pCR were evaluated using the $\chi 2$ test or Fisher's exact test, when appropriate. To take into account the effects of all variables on pCR, multivariate analysis were performed by a multivariate logistic regression to estimate the adjuster Odds Ratios (ORs). Statistical significance was set at $p<0.05$. All analyses were performed using SAS 9.4 (SAS Institute Inc., Cary, NC, USA).

\section{Results}

Clinicopathological features of the 54 patients are detailed in Table 1 . Briefly, the mean age at diagnosis was 50 years (range $28-75$ ). In $87 \%$ of cases the pre-NACT tumor diameter 
TABLE 1: Clinicopathological features of the study population.

\begin{tabular}{|c|c|}
\hline Characteristics & $\begin{array}{c}\text { Number of } \\
\text { patients (\%) }\end{array}$ \\
\hline \multicolumn{2}{|l|}{ Age (y) } \\
\hline$\leq 50$ & $30(55 \%)$ \\
\hline$>50$ & $24(45 \%)$ \\
\hline \multicolumn{2}{|l|}{ Pre-NACT tumor size (cT) } \\
\hline$\leq 2 \mathrm{~cm}$ & $7(13 \%)$ \\
\hline$>2 \mathrm{~cm}$ & $47(87 \%)$ \\
\hline \multicolumn{2}{|l|}{ Pre-NACT nodal status $(\mathrm{cN})$} \\
\hline Positive & $24(45 \%)$ \\
\hline Negative & $30(55 \%)$ \\
\hline \multicolumn{2}{|l|}{ Histotype } \\
\hline Ductal & $51(94 \%)$ \\
\hline Lobular & $1(2 \%)$ \\
\hline Others & $2(4 \%)$ \\
\hline \multicolumn{2}{|l|}{ Nuclear grade } \\
\hline Grades 1-2 & 0 \\
\hline Grade 3 & $54(100 \%)$ \\
\hline \multicolumn{2}{|l|}{ Ki-67 } \\
\hline$<50 \%$ & $14(26 \%)$ \\
\hline$\geq 50 \%$ & $40(74 \%)$ \\
\hline \multicolumn{2}{|l|}{ Post-NACT surgery } \\
\hline Mastectomy & $30(55 \%)$ \\
\hline Segmental mastectomy & $24(45 \%)$ \\
\hline Complete pathological response (pCR) to NACT & $19(35 \%)$ \\
\hline
\end{tabular}

was larger than $2 \mathrm{~cm}$. Axillary node involvement, assessed by echography and confirmed by fine needle aspiration cytology, was present in 24 patients (45\%). The most common histologic subtype was ductal carcinoma of no special type (94\% of cases). All tumors were of high nuclear grade (G3) with a proliferation index $\geq 50 \%$ in the large majority of cases (74\%).

After NACT 30 patients (55\%) underwent mastectomy and 24 (45\%) had conservative breast surgery, A pCR was achieved in 19 patients (35\%).

3.1. Tumor-Infiltrating Lymphocytes and Immunophenotype of the Inflammatory Infiltrates in Pre-NACT Biopsies. The results of stromal TILs evaluations are detailed in Table 2. Briefly, stromal TILs were present in 51 pre-NACT biopsies (95\%), with percentages ranging from 2 to $80 \%$ (Figures 1(a) and $1(\mathrm{~b}))$. Twenty-four cases (45.5\%) had $50 \%$ or greater stromal TILs (high TILs) and were accordingly classified as lymphocyte predominant breast cancer (LPBC) [13]. Immunophenotyping of the inflammatory infiltrates revealed a predominance of CD3+ T cells in all biopsy samples. In 36 biopsies $(70 \%)$ the most represented was the CD8+ subset, followed by the CD4+ subset (27\%). Intriguingly, NK cells were absent in $96 \%$ of biopsies.
3.2. Expression of PD-L1 on Neoplastic and Inflammatory Cells. Membrane staining of neoplastic cells was present in 19 preNACT biopsies (35\%), with an extent ranging from 1 to $90 \%$ (Figures 1(c) and 1(d)). In over 95\% of these biopsies (18/19) the percentage of $\mathrm{PD}-\mathrm{L} 1$ positive tumor cells did not exceed $50 \%$. The membrane stain scored $3+$ in $8 / 19$ cases $(42 \%)$, $2+$ in $5 / 19(26 \%)$, and $1+$ in $6 / 19$ (32\%). PD-L1 staining of inflammatory cells was present in the majority of biopsies (44 cases, $81 \%$ ) (Figures 1(e) and 1(f)).

Univariate analysis showed a significant association between the presence of high stromal TILS and the expression of PD-L1 on $\geq 25 \%$ of tumor cells $(p=0.008)$ and $\geq 10 \%$ of inflammatory cells $(p=0.002)$; this association was independent from the prevalent lymphocyte subset (CD8+ or $\mathrm{CD} 4+$ ), and from the CD8/ CD4 ratio.

3.3. Comparison of Histologic and Immunohistochemical Data with the Response to NACT. A pCR was achieved in 19 patients (35\%). Univariate analysis (Table 2) showed a significant association between the expression of PD-L1 in $\geq 25 \%$ of neoplastic cells and pCR $(p=0.02)$. The presence of pCR was also significantly more frequent in cases showing features of LPBC (with high TILs) in the pre-NACT biopsies $(p<0.001)$. Moreover, pCR was achieved in $100 \%$ of patients showing both high TILs and expression levels of PD-L1 $\geq$ $25 \%$ in neoplastic cells in the pre-NACT biopsies $(p=0.011$, Table 3).

At multivariate analysis (Table 4), only PD-L1 expression on tumor cells remained significantly associated with pCR ( $p=0.038)$ with OR of 1,13 (95\% CI 1,01-1,27).

\section{Discussion}

The immune system is strongly involved both in the tumor surveillance and in the pathogenesis of breast cancer. Moreover, preexisting immunity against tumor cells is a crucial factor that influences the response to chemotherapy. It is now believed that preexisting antitumor immunity is activated or enhanced during the initial cycle of chemotherapy. During the subsequent cycles, together with incoming acquired drug resistance of the tumor cells, the onset of immune resistance mechanisms impairs the efficacy of treatment $[19,20]$.

Due to the high histological grading and mutational load, along with the activation of large amounts of genes implicated in immune function, TNBC seems to be the subtype more likely associated with immune system involvement. Thus, the identification of novel immunological prognostic and predictive biomarkers would be useful to guide the choice of the most appropriate treatment, as well as the optimal timing of surgery, especially in the neoadjuvant setting.

Either the presence of stromal TILs or the expression of PD-L1 is being actively investigated as prognostic biomarkers in TNBC. This subset of breast cancer has an aggressive clinical and biological behaviour, with higher risk for early recurrences and a poorer prognosis as compared to the other BC subtypes. However, in the neoadjuvant setting, the achievement of a pCR after NACT is associated with longterm survival. In this study, we investigated the hypothesis that the presence of high stromal TILs and the expression 


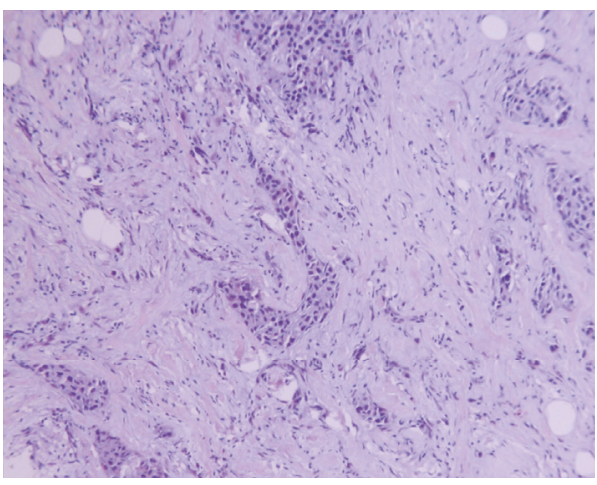

(a)

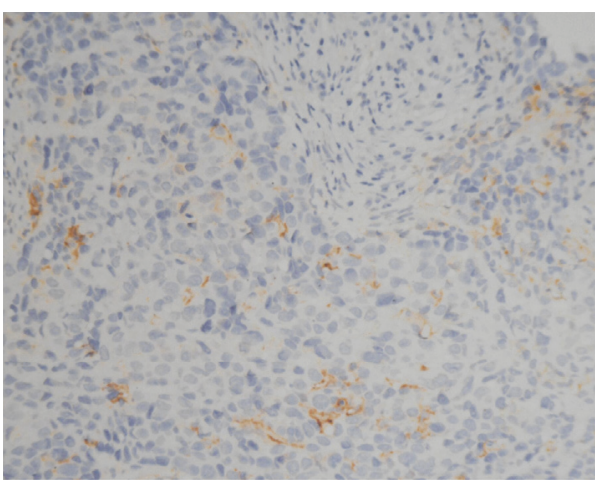

(c)

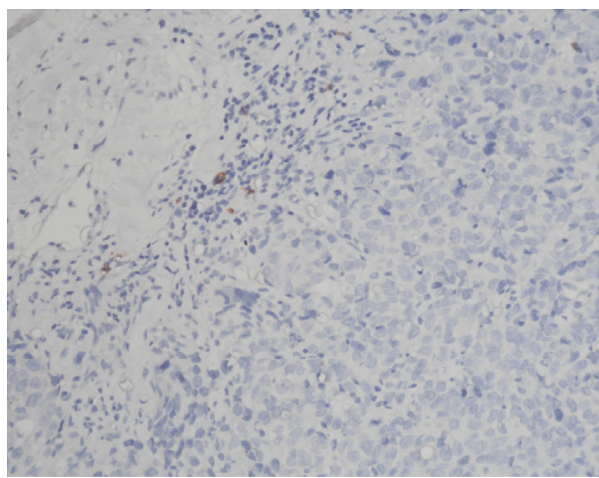

(e)

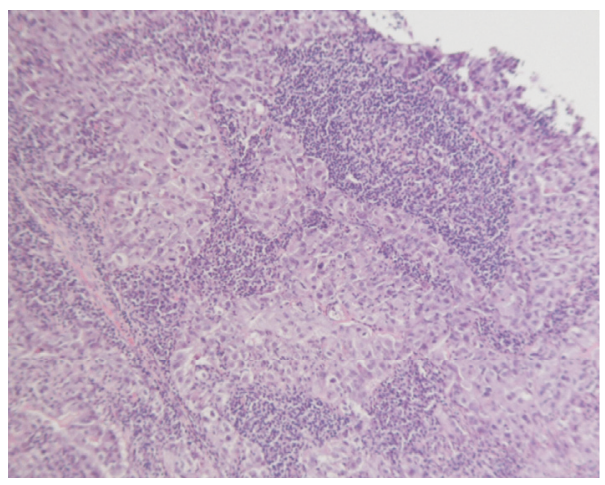

(b)

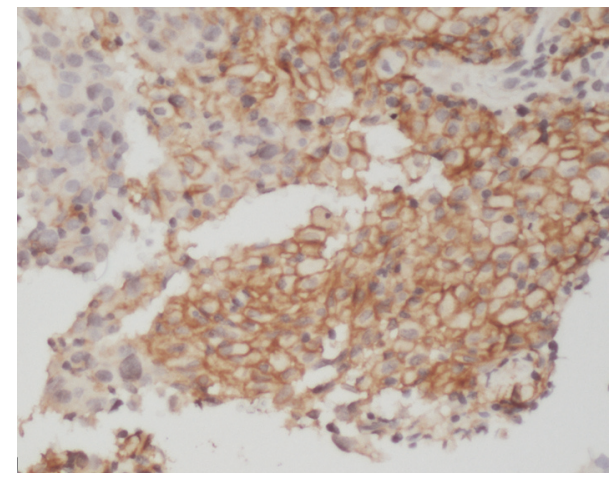

(d)

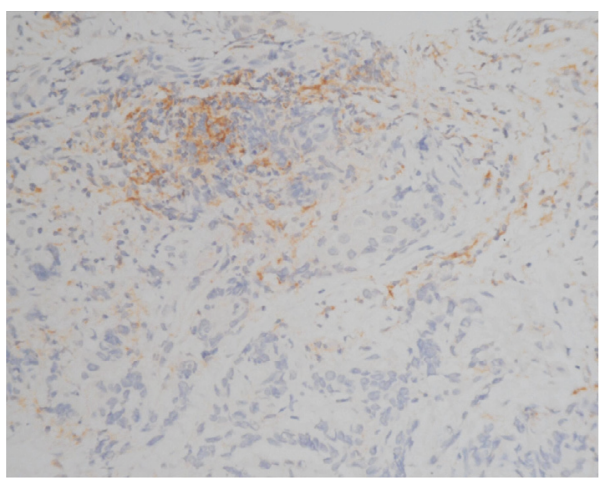

(f)

Figure 1: Evaluation of stromal TILs and PD-L1 expression in TNBC core biopsies. (a)-(b): low (a) and high (b) level of stromal tumorinfiltrating lymphocytes (haematoxylin and eosin, original magnification $\times 10)$. (c)-(d): membranous PD-L1 stain in scattered (c) and diffuse neoplastic cells (d) (PD-L1 immunohistochemical stain, original magnification $\times 20)$. (e)-(f): membranous PD-L1 stain in scattered (e) and diffuse inflammatory cells (f) (PD-L1 immunohistochemical stain, original magnification $\times 20)$.

of PD-L1, both markers of immune activation in the tumor microenvironment, could be associated with the rate of $\mathrm{pCR}$ in TNBC.

We evaluated pre-NACT core biopsies, which proved to be qualitatively and quantitatively adequate for our analysis. In our study population, $35 \%$ of patients achieved a pCR, which is in line with recently published literature $[21,22]$.

On microscopic evaluation of pre-NACT core biopsies, tumor cell expression of PD-L1 was observed in 35\% of cases, although at low levels $(\geq 1 \%<25 \%$ in $15 / 19$ biopsies, $79 \%)$. Our observation on a pure sample of TNBC confirms the results of Dill et al. [16] who analyzed a large number of BC with various histologic subtypes, showing the highest rate of PD-L1 expression (32\%) in TNBC, with only $5 \%$ with diffuse expression on tumor cells $(>50 \%)$.

PD-L1 expression, both on neoplastic and inflammatory cells, was significantly associated with high stromal TILs. Our observation extends the results reported by Mori et al. [23], which showed a significant association between PDL1 expression on tumor cells and percentage of stromal TILs on surgical breast specimens, and confirms the parallel behaviour of these immune biomarkers in TNBC. The limited 
TABLE 2: Association between stromal TILs, the expression of PD-L1 on tumor cells and inflammatory infiltrate, Ki-67 value, cT, cN, and pCR in the univariate analysis.

\begin{tabular}{|c|c|c|c|}
\hline & \multirow{2}{*}{$N(\%)$} & \multicolumn{2}{|c|}{$\mathrm{pCR}$} \\
\hline & & $N^{\circ}$ & $p$ value \\
\hline \multicolumn{4}{|l|}{ Stromal TILs } \\
\hline Absent/low & $32(59 \%)$ & 8 & \multirow{2}{*}{$<0.001$} \\
\hline High & $22(41 \%)$ & 11 & \\
\hline \multicolumn{4}{|c|}{ PD-L1 on tumor cells } \\
\hline $0 \%$ & $35(65 \%)$ & 11 & \multirow[b]{3}{*}{0.024} \\
\hline$\geq 1-<25 \%$ & $15(28 \%)$ & 4 & \\
\hline$\geq 25 \%$ & $4(7 \%)$ & 4 & \\
\hline \multicolumn{4}{|c|}{ PD-L1 on inflammatory cells } \\
\hline Negative & $10(19 \%)$ & 3 & \multirow{2}{*}{$n s$} \\
\hline Positive & $44(81 \%)$ & 15 & \\
\hline \multicolumn{4}{|l|}{$\mathrm{Ki}-67$} \\
\hline$<50 \%$ & $14(26 \%)$ & 4 & \multirow{2}{*}{ ns } \\
\hline$\geq 50 \%$ & $40(74 \%)$ & 15 & \\
\hline \multicolumn{4}{|l|}{$\mathrm{cT}$} \\
\hline $\mathrm{T} 1$ & $7(13 \%)$ & 3 & \multirow{2}{*}{$n s$} \\
\hline $\mathrm{T} 2-\mathrm{T} 4$ & $47(87 \%)$ & 16 & \\
\hline \multicolumn{4}{|l|}{$\mathrm{cN}$} \\
\hline Negative & $30(56 \%)$ & 13 & \multirow{2}{*}{$n s$} \\
\hline Positive & $24(44 \%)$ & 6 & \\
\hline
\end{tabular}

TABLE 3: The achievement of pCR according to levels of both stromal TILs and PD-L1 expression on neoplastic cell membranes (low TILs/low PD-L1; high TILs/low PD-L1; low TILs/high PD-L1; high TILs/high PD-L1).

\begin{tabular}{lllcccc}
\hline \multicolumn{6}{c}{ TILs } & \multicolumn{5}{c}{ PD-L1 on neoplastic cells } \\
& pt & pt & $p C 25 \%$ & pt & $p C R$ & \\
\hline Low & 32 & 32 & $25 \%$ & 0 & & $p=0.011$ \\
High & 22 & 18 & $39 \%$ & 4 & $100 \%$ & \\
\hline
\end{tabular}

amount of published reports in pure cohorts of TNBC seems to suggest a favorable prognostic role of $\mathrm{PD}-\mathrm{L} 1$, despite some discrepancies. Mori et al. [23] demonstrated that the interaction between TILs and PD-L1 correlates with a better clinical outcome. However, when high PD-L1 expression is associated with low levels of stromal TILs the prognosis is poor [24]. In the study of Beckers et al. [25] PD-L1, although associated with a better outcome, failed to show an independent prognostic role in this subset of tumors. These partial discrepancies could be explained by differences in the choice of clinical outcomes, in the methods of evaluation of PD-L1 expression on neoplastic cells (membranous versus cytoplasmic) and the cut-off values adopted, and in the antibodies used and the type of sample evaluated (core biopsies versus surgical samples).

There are only limited data on the predictive value of these two biomarkers in TNBC. We found that in this breast cancer subtype the concomitant expression of stromal TILs and PDL1 on tumor cells membranes was significantly associated with pCR. According to our results, a cut-off of PD-L1 membrane expression on $\geq 25 \%$ of neoplastic cells in preneoadjuvant biopsies predicted pCR for TNBC, regardless of staining intensity. On the contrary, the predictive role of TILs showed only a limited power and no statistical association on multivariate analysis. In light of the preliminary results of the KEYNOTE 173 phase II trial [26], reporting a $90 \%$ pCR rate in TNBC treated in this setting with the adjunct of pembrolizumab to standard chemotherapy, we hypothesize that TNBC expressing PD-L1 in less than 25\% of tumor cells could represent the subset most likely to benefit from this association.

Immunophenotyping of tumor inflammatory microenvironment revealed an excess of $\mathrm{CD} 8$ + with a ratio of CD8/CD4 $>1$, in line with previous reports $[27,28]$, although this observation did not reach statistical significance probably due to our limited sample size. Additionally, we found negligible amounts of NK cells in pre-NACT biopsies, although we cannot exclude the fact that their level could have been increased after the first cycle of chemotherapy due to tumor cells death and the release tumor associated antigens.

In conclusion, we showed that a cut-off value of PD-L1 in $\geq 25 \%$ of tumor cells predicts pCR in TNBC and to our knowledge our study is the first dealing with an exclusive population of TNBC cases. A possible explanation for our observation is that PD-L1 expression could be associated with a subpopulation of TNBC with a more aggressive behaviour, 
TABLE 4: Association between the expression of PD-L1 on neoplastic cells and inflammatory infiltrate, stromal TILs, Ki67, clinical T, clinical $\mathrm{N}$, and pCR in the multivariate analysis.

\begin{tabular}{|c|c|c|c|c|}
\hline & \multirow{2}{*}{$N(\%)$} & \multicolumn{3}{|c|}{ pCR } \\
\hline & & $N(\%)$ & $p$ value & ORR (CI) \\
\hline \multicolumn{5}{|c|}{ Stromal TILs } \\
\hline Low & $32(60 \%)$ & $8(25 \%)$ & \multirow{2}{*}{0.5} & \multirow{2}{*}{$1,61(0,40-6,52)$} \\
\hline High & $22(40 \%)$ & $11(50 \%)$ & & \\
\hline \multicolumn{5}{|c|}{ PD-L1 on tumor cells } \\
\hline $0 \%$ & $35(65 \%)$ & $11(31 \%)$ & \multirow{3}{*}{0.038} & \multirow{3}{*}{$1,13(1,01-1,27)$} \\
\hline $1-25 \%$ & $15(28 \%)$ & $4(27 \%)$ & & \\
\hline$\geq 25 \%$ & $4(7 \%)$ & $4(100 \%)$ & & \\
\hline \multicolumn{5}{|c|}{ PD-L1 on inflammatory cells } \\
\hline Negative & $10(18 \%)$ & $3(30 \%)$ & \multirow{2}{*}{0.058} & \multirow{2}{*}{$0,09(0,01-1,08)$} \\
\hline Positive & $44(82 \%)$ & $15(34 \%)$ & & \\
\hline \multicolumn{5}{|l|}{ Ki-67 } \\
\hline$<50 \%$ & $14(26 \%)$ & $4(28 \%)$ & \multirow{2}{*}{0.054} & \multirow{2}{*}{$1,05(1-1,09)$} \\
\hline$\geq 50 \%$ & $40(74 \%)$ & $15(37 \%)$ & & \\
\hline \multicolumn{5}{|l|}{ Clinical T } \\
\hline $\mathrm{T} 1$ & $7(13 \%)$ & $3(43 \%)$ & \multirow{2}{*}{0.8} & \multirow{2}{*}{$0,8(0,08-8,09)$} \\
\hline $\mathrm{T} 2-\mathrm{T} 4$ & $47(87 \%)$ & $16(34 \%)$ & & \\
\hline \multicolumn{5}{|l|}{ Clinical N } \\
\hline Negative & $30(55 \%)$ & $13(43 \%)$ & \multirow{2}{*}{0.27} & \multirow{2}{*}{$0,47(0,12-1,82)$} \\
\hline Positive & $24(45 \%)$ & $6(25 \%)$ & & \\
\hline
\end{tabular}

likely to respond to chemotherapy. Further studies with larger number of cases are warranted to confirm our findings.

\section{Consent}

Informed consent was waived from the Ethical Committee.

\section{Disclosure}

The preliminary results of this work were presented as a poster at the ESMO 2017 Congress in Madrid, Spain.

\section{Conflicts of Interest}

The authors declare no potential conflicts of interest with respect to the research, authorship, and/or publication of this article.

\section{Acknowledgments}

The authors thank Dr. Elisa Concetta Onesti for her help in the analysis of data and in the preparation of the abstract for ESMO.

\section{References}

[1] P. Boyle, "Triple-negative breast cancer: epidemiological considerations and recommendations," Annals of Oncology, vol. 23, Supplement 6, Article ID mds187, pp. vi7-vi12, 2012.

[2] P. Rastogi, S. J. Anderson, H. D. Bear et al., "Preoperative chemotherapy: Updates of national surgical adjuvant breast and bowel project protocols B-18 and B-27," Journal of Clinical Oncology, vol. 26, no. 5, pp. 778-785, 2008.

[3] V. Guarneri, K. Broglio, S. Kau et al., "Prognostic Value of Pathologic Complete Response After Primary Chemotherapy in Relation to Hormone Receptor Status and Other Factors," Journal of Clinical Oncology, vol. 24, no. 7, pp. 1037-1044, 2006.

[4] W. D. Foulkes, I. E. Smith, and J. S. Reis-Filho, "Triple-negative breast cancer," The New England Journal of Medicine, vol. 363, no. 20, pp. 1938-1948, 2010.

[5] M. Ono, H. Tsuda, C. Shimizu et al., "Tumor infiltrating lymphocytes are correlated with response to neoadjuvant chemotherapy in triple-negative breast cancer," Breast Cancer Research and Treatment, vol. 132, no. 3, pp. 793-805, 2012.

[6] R. Yamaguchi, M. Tanaka, A. Yano et al., “Tumor-infiltrating lymphocytes are important pathologic predictors for neoadjuvant chemotherapy in patients with breast cancer," Human Pathology, vol. 43, no. 10, pp. 1688-1694, 2012.

[7] C. Denkert, G. von Minckwitz, J. C. Brase et al., "TumorInfiltrating Lymphocytes and Response to Neoadjuvant Chemotherapy With or Without Carboplatin in Human Epidermal Growth Factor Receptor 2-Positive and TripleNegative Primary Breast Cancers," Journal of Clinical Oncology, vol. 33, no. 9, pp. 983-991, 2015.

[8] G. Pruneri, A. Vingiani, V. Bagnardi et al., "Clinical validity of tumor-infiltrating lymphocytes analysis in patients with triplenegative breast cancer," Annals of Oncology, vol. 27, no. 2, pp. 249-256, 2016.

[9] M. Ilie, V. Hofman, M. Dietel, J. Soria, and P. Hofman, "Assessment of the PD-L1 status by immunohistochemistry: challenges and perspectives for therapeutic strategies in lung cancer patients," Virchows Archiv, vol. 468, no. 5, pp. 511-525, 2016. 
[10] M. J. Butte, V. Peña-Cruz, M.-J. Kim, G. J. Freeman, and A. H. Sharpe, "Interaction of human PD-L1 and B7-1," Molecular Immunology, vol. 45, no. 13, pp. 3567-3572, 2008.

[11] S. Muenst, A. R. Schaerli, F. Gao et al., "Expression of programmed death ligand 1 (PD-L1) is associated with poor prognosis in human breast cancer," Breast Cancer Research and Treatment, vol. 146, no. 1, pp. 15-24, 2014.

[12] K. A. Schalper, V. Velcheti, D. Carvajal et al., "In Situ Tumor PD-L1 mRNA Expression Is Associated with Increased TILs and Better Outcome in Breast Carcinomas," Clinical Cancer Research, vol. 20, no. 10, pp. 2773-2782, 2014.

[13] R. Salgado, C. Denkert, S. Demaria et al., "The evaluation of tumor-infiltrating lymphocytes (TILs) in breast cancer: recommendations by an International TILs Working Group 2014," Annals of Oncology, vol. 26, no. 2, pp. 259-271, 2015.

[14] F. R. Hirsch, A. McElhinny, D. Stanforth et al., "PD-L1 immunohistochemistry assays for lung cancer: results from phase 1 of the blueprint PD-L1 IHC assay comparison project," Journal of Thoracic Oncology, vol. 12, no. 2, pp. 208-222, 2017.

[15] A. Rittmeyer, F. Barlesi, D. Waterkamp et al., "Atezolizumab versus docetaxel in patients with previously treated non-smallcell lung cancer (OAK): a phase 3, open-label, multicentre randomised controlled trial," The Lancet, vol. 389, no. 10066, pp. 255-265, 2017.

[16] E. A. Dill, A. A. Gru, K. A. Atkins et al., "PD-L1 Expression and Intratumoral Heterogeneity Across Breast Cancer Subtypes and Stages," The American Journal of Surgical Pathology, vol. 41, no. 3, pp. 334-342, 2017.

[17] W. F. Symmans, F. Peintinger, C. Hatzis et al., "Measurement of residual breast cancer burden to predict survival after neoadjuvant chemotherapy," Journal of Clinical Oncology, vol. 25, no. 28, pp. 4414-4422, 2007.

[18] http://www3.mdanderson.org/app/medcalc/index.cfm?pagename $=$ jsconvert3, 2007.

[19] F. Ghiringhelli and L. Apetoh, "The interplay between the immune system and chemotherapy: emerging methods for optimizing therapy," Expert Review of Clinical Immunology, vol. 10, no. 1, pp. 19-30, 2013.

[20] S. Demaria, M. D. Volm, R. L. Shapiro et al., "Development of tumor-infiltrating lymphocytes in breast cancer after neoadjuvant paclitaxel chemotherapy," Clinical Cancer Research, vol. 7, pp. 3025-3030, 2001.

[21] T. Gamucci, L. Pizzuti, I. Sperduti et al., "Neoadjuvant chemotherapy in triple-negative breast cancer: A multicentric retrospective observational study in real-life setting," Journal of Cellular Physiology, 2017.

[22] Z. Shao, S. Chaudhri, M. Guo, L. Zhang, and D. Rea, "Neoadjuvant Chemotherapy in Triple Negative Breast Cancer: An Observational Study," Oncology Research : Featuring Preclinical and Clinical Cancer Therapeutics, vol. 23, no. 6, pp. 291-302, 2016.

[23] H. Mori, M. Kubo, R. Yamaguchi et al., "The combination of PDL1 expression and decreased tumor-infiltrating lymphocytes is associated with a poor prognosis in triple-negative breast cancer," Oncotarget, vol. 8, no. 9, pp. 15584-15592, 2017.

[24] N. Tomioka, M. Azuma, M. Ikarashi et al., "The therapeutic candidate for immune checkpoint inhibitors elucidated by the status of tumor-infiltrating lymphocytes (TILs) and programmed death ligand 1 (PD-L1) expression in triple negative breast cancer (TNBC)," Breast Cancer, 2017.

[25] R. K. Beckers, C. I. Selinger, R. Vilain et al., "Programmed death ligand 1 expression in triple-negative breast cancer is associated with tumour-infiltrating lymphocytes and improved outcome," Histopathology, vol. 69, no. 1, pp. 25-34, 2016.

[26] R. Nanda, L. Q. M. Chow, E. C. Dees et al., "Pembrolizumab in patients with advanced triple-negative breast cancer: phase Ib KEYNOTE-012 Study," Journal of Clinical Oncology, vol. 34, pp. 2460-2467, 2016.

[27] S. M. A. Mahmoud, E. C. Paish, D. G. Powe et al., "Tumorinfiltrating $\mathrm{CD}^{+}$lymphocytes predict clinical outcome in breast cancer," Journal of Clinical Oncology, vol. 29, no. 15, pp. 1949-1955, 2011.

[28] A. N. Seo, H. J. Lee, E. J. Kim et al., "Tumour-infiltrating CD8+ lymphocytes as an independent predictive factor for pathological complete response to primary systemic therapy in breast cancer," British Journal of Cancer, vol. 109, no. 10, pp. 2705-2713, 2013. 


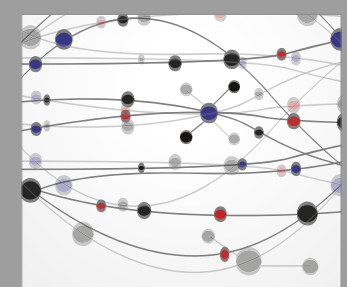

The Scientific World Journal
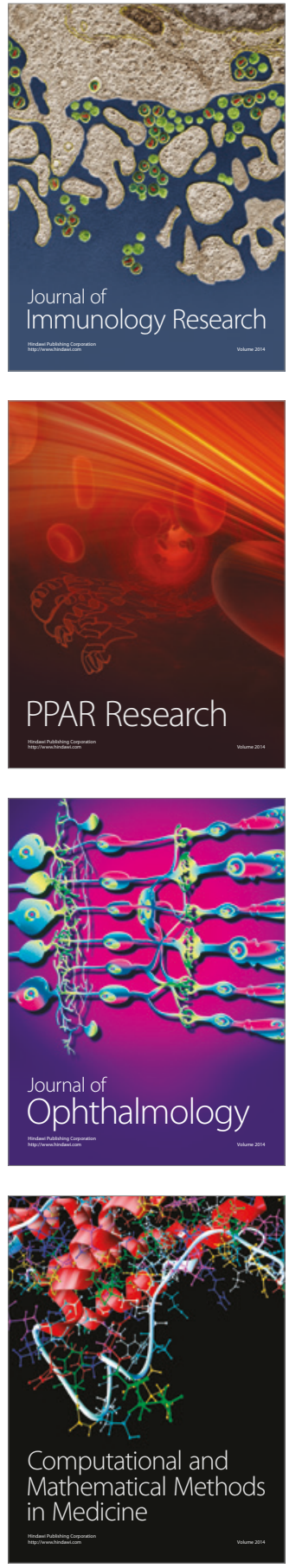

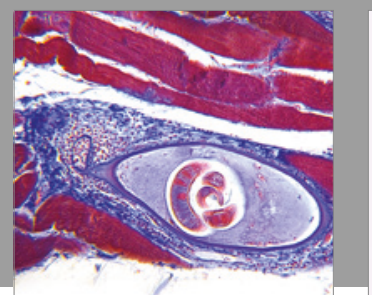

Gastroenterology Research and Practice
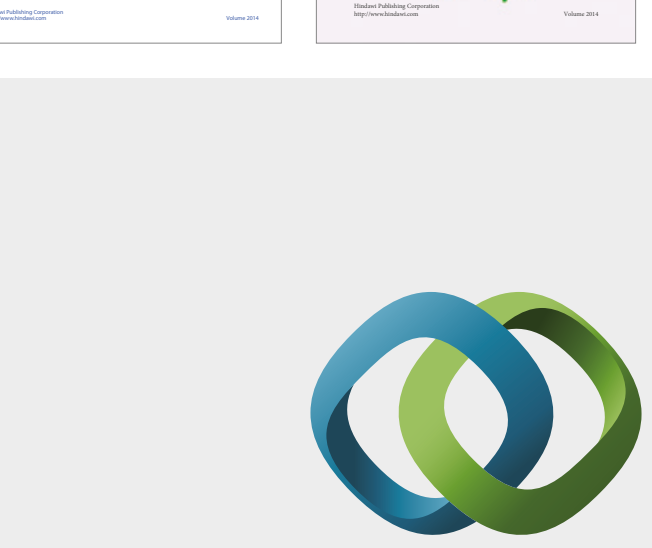

\section{Hindawi}

Submit your manuscripts at

https://www.hindawi.com
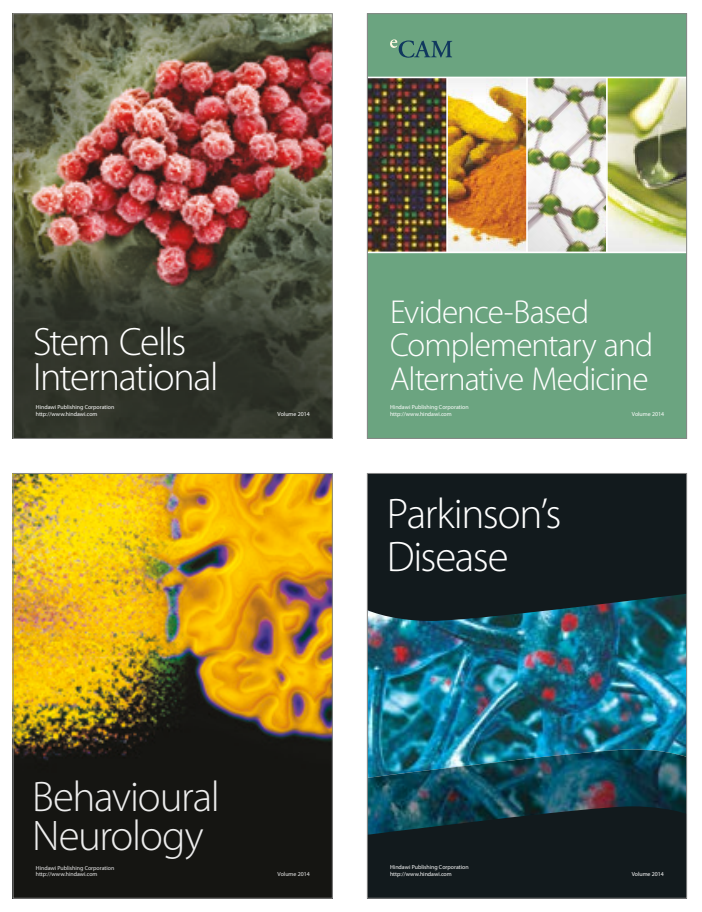
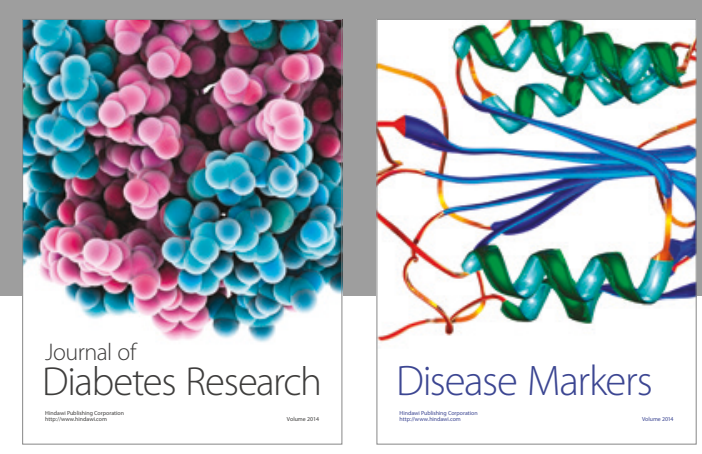

Disease Markers
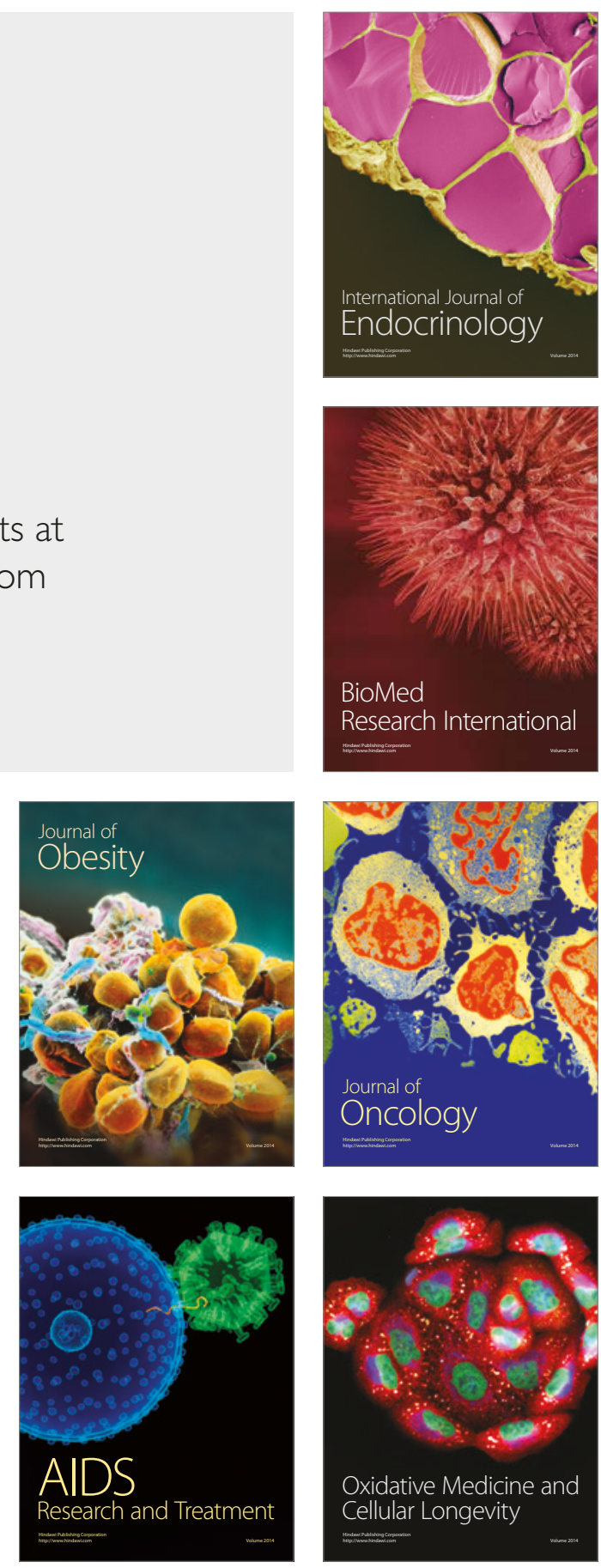